\title{
Nuevo apero para la realización de microcuencas de captación de escorrentía para la restauración forestal
}

\author{
Jorge Mongil Manso* \\ Grupo de Hidrología y Conservación de Aguas y Suelos. Universidad Católica de Ávila. Canteros s/n 05005-Ávila \\ *Autor para correspondencia: jorge.mongil@ucavila.es
}

\section{Resumen}

Se describe un nuevo apero (Saltusaqua) (patente ES2485115B1) diseñado para la realización mecanizada de microcuencas semicirculares de captación de escorrentía, de utilidad en restauración forestal y plantaciones de árboles en general. Se trata de un apero formado por tres planchas rectangulares - una principal y dos laterales- de acero o material adecuado, unidas formando la principal con cada una de las laterales un ángulo de $135^{\circ}$. La plancha principal y las laterales tienen acoplada una cuchilla en la parte inferior. El cuerpo del apero tiene adosado un sistema de enganche, que será diferente en el caso de que se quiera acoplar a un bulldózer o a un tractor agrícola. Se ha utilizado el modelo Modipé para evaluar la preparación del suelo y la unidad sistematizada que crea el apero diseñado. Del análisis se puede concluir que, a pesar de tratarse de una sistematización primaria incompleta, con el caballón semicircular se consigue recoger aproximadamente un $125 \%$ más de agua que en la ladera original y un $74 \%$ más que la lluvia caída. Por ello, la preparación del suelo realizada se considera muy eficiente desde el punto de vista de la captación de agua. Frente a una preparación manual, este apero realiza microcuencas semicirculares de manera mecanizada y económica. El aporte suplementario de agua que supone la captación de lluvia y escorrentía podría favorecer la supervivencia y el desarrollo de las plantas que se sitúan en las microcuencas.

Palabras clave: caballón semicircular, Modipé, oasificación, recolección de agua, repoblación forestal. 


\section{Introducción}

La plantación de árboles y arbustos en zonas áridas y semiáridas ha sido siempre un reto para el hombre. Para que tenga éxito se requiere una preparación previa del terreno, que garantice la satisfacción de las necesidades hídricas de las plantas instaladas en sus primeros años de desarrollo, que suelen ser los más críticos. La preparación pretende mejorar las condiciones del suelo para favorecer la instalación de los pequeños árboles recién implantados. Así, en muchos lugares se desarrollaron técnicas de recolección de agua, para aplicarlas a este propósito dentro del ámbito agrícola primero y de la repoblación forestal después. No obstante, estas técnicas, que consisten en la remoción de volúmenes de tierra más o menos elevados, se vienen realizando, salvo algunas excepciones, de forma manual, lo que resulta excesivamente gravoso.

Los caballones semicirculares son bancales de tierra de forma semicircular con los extremos situados sobre la misma curva de nivel, tradicionalmente de 2 a $20 \mathrm{~m}$ de radio. Estos caballones -también llamados medias-lunas, demi-lunes en los países francófonos africanos y bordos semicirculares en Latinoamérica- se emplean para la plantación de árboles y arbustos y, en algunos casos, también para cultivos. En Ouramiza (Níger), por ejemplo, se han construido medias-lunas con un caballón de 20 $\mathrm{cm}$ de altura, $6 \mathrm{~m}^{2}$ de superficie cultivada y $16 \mathrm{~m}^{2}$ de área de impluvio. Las mediaslunas tienen un radio de $2 \mathrm{~m}$, la distancia entre estructuras dentro de la misma línea

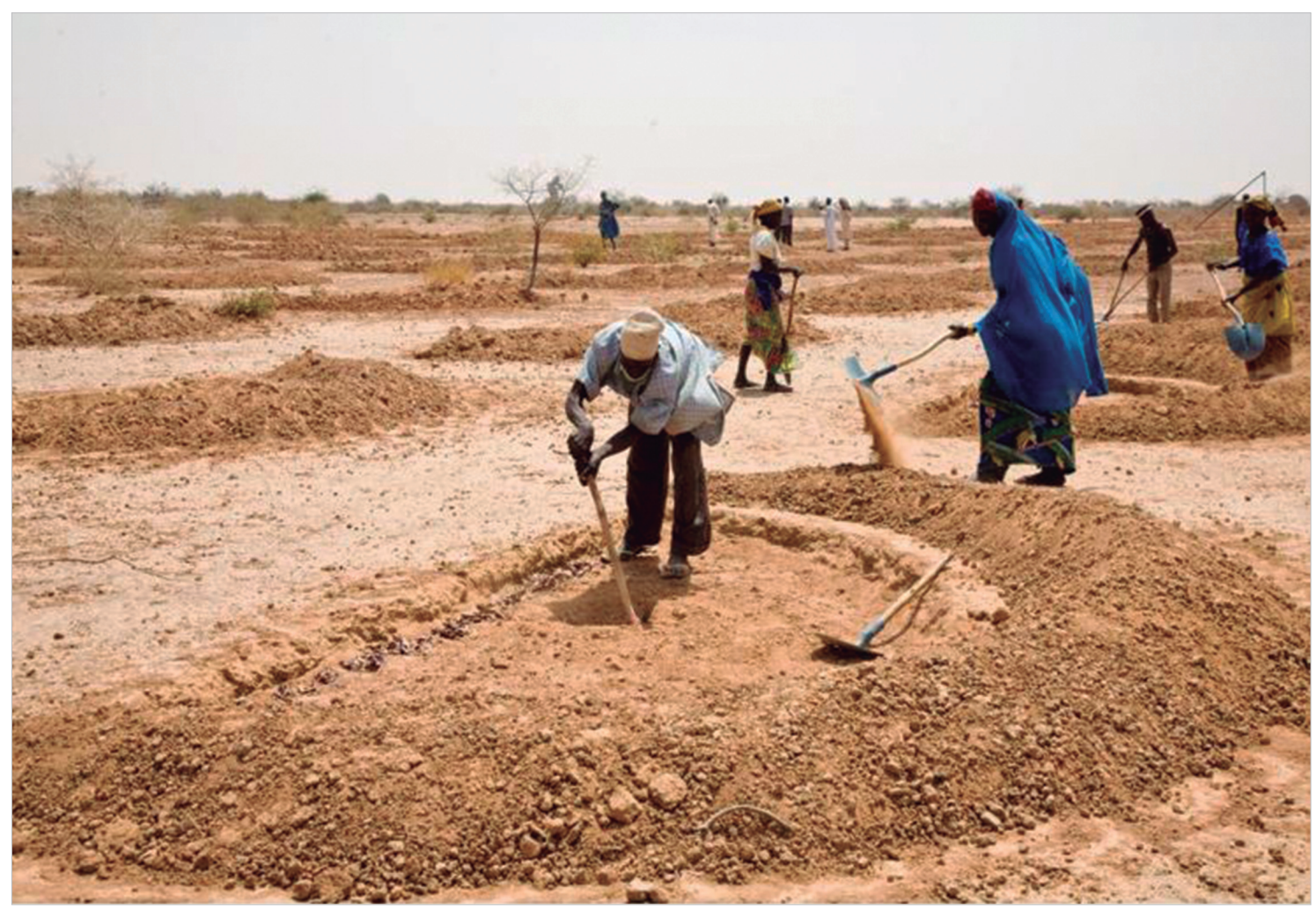

Figura 1. Construcción manual de caballones semicirculares en Níger (Fotografía: www.oxfam.org). 
es de 4 m, idéntica a la distancia de separación entre líneas (Roose, 1994) (Fig. 1). Las microcuencas, que recogen agua de lluvia y escorrentía para que la planta disponga de una mayor humedad, vienen haciéndose tradicionalmente de forma manual, debido a la inexistencia de aperos adecuados para su realización mecanizada. Esto hace que los costes se eleven o que no puedan realizarse las microcuencas por falta de mano de obra. Por esta razón es importante desarrollar un apero que sea capaz de obtener microcuencas mecanizadamente y a costes reducidos.

\section{Objetivos}

El objetivo de este trabajo es presentar un nuevo apero (Saltusaqua) (patente ES2485115B1) que, acoplado a un tractor agrícola o forestal o a un bulldózer, es capaz de perfilar microcuencas semicirculares para la captación de escorrentía superficial, que permite el establecimiento de restauraciones forestales, cultivos leñosos o plantaciones de árboles en general, en zonas secas.

\section{Descripción detallada del apero}

El apero es capaz de realizar microcuencas de forma semicircular, de tamaño variable. Se trata de un apero (Fig. 2 y 3 ) formado por tres planchas rectangulares -una principal y dos laterales- de acero de $10 \mathrm{~mm}$ de espesor, soldadas formando, la principal con cada una de las laterales, un ángulo de $135^{\circ}$. Estas tres planchas llevan acopladas mediante tornillos unas cuchillas recambiables, en la parte inferior, formando un ángulo de $20^{\circ}$. Para dar estabilidad al conjunto, se suelda otra plancha de acero denominada plancha de sujeción y refuerzo, y un chasis de tubos de acero de sección rectangular en posición horizontal y vertical. Se han diseñado dos aperos de dimensiones diferentes (Tab. 1) (modelos a y b), para realizar microcuencas de diferentes anchuras.

El cuerpo del apero tiene adosados dos sistemas de enganche para acoplarlo a un bulldózer o a un tractor agrícola. El enganche al bulldózer se realiza a través del subsolador o dispositivo porta-rejones de esta máquina. Una barra de acero, de dimensiones similares a las de los rejones de un subsolador, se suelda al cuerpo principal del apero. Esta barra se engancha al subsolador del bulldózer mediante un bulón. El subsolador tiene capacidad para elevar o bajar el apero mediante un sistema de accionamiento hidráulico. El acoplamiento a un tractor agrícola se realiza mediante el denominado enganche tripuntal, que consiste en tres brazos situados en la parte posterior del tractor (dos actuadores y un tensor), mediante el cual el apero puede levantarse y bajarse (el enganche debe de ser doble efecto), accionado por el motor del tractor. Para poder acoplar el apero al tractor agrícola, por consiguiente, se sueldan en la parte anterior de la plancha principal del apero dos pares de planchas de acero perforadas (tiros) y un par de planchas más (torretas) se unen a la barra de enganche al bulldózer. 

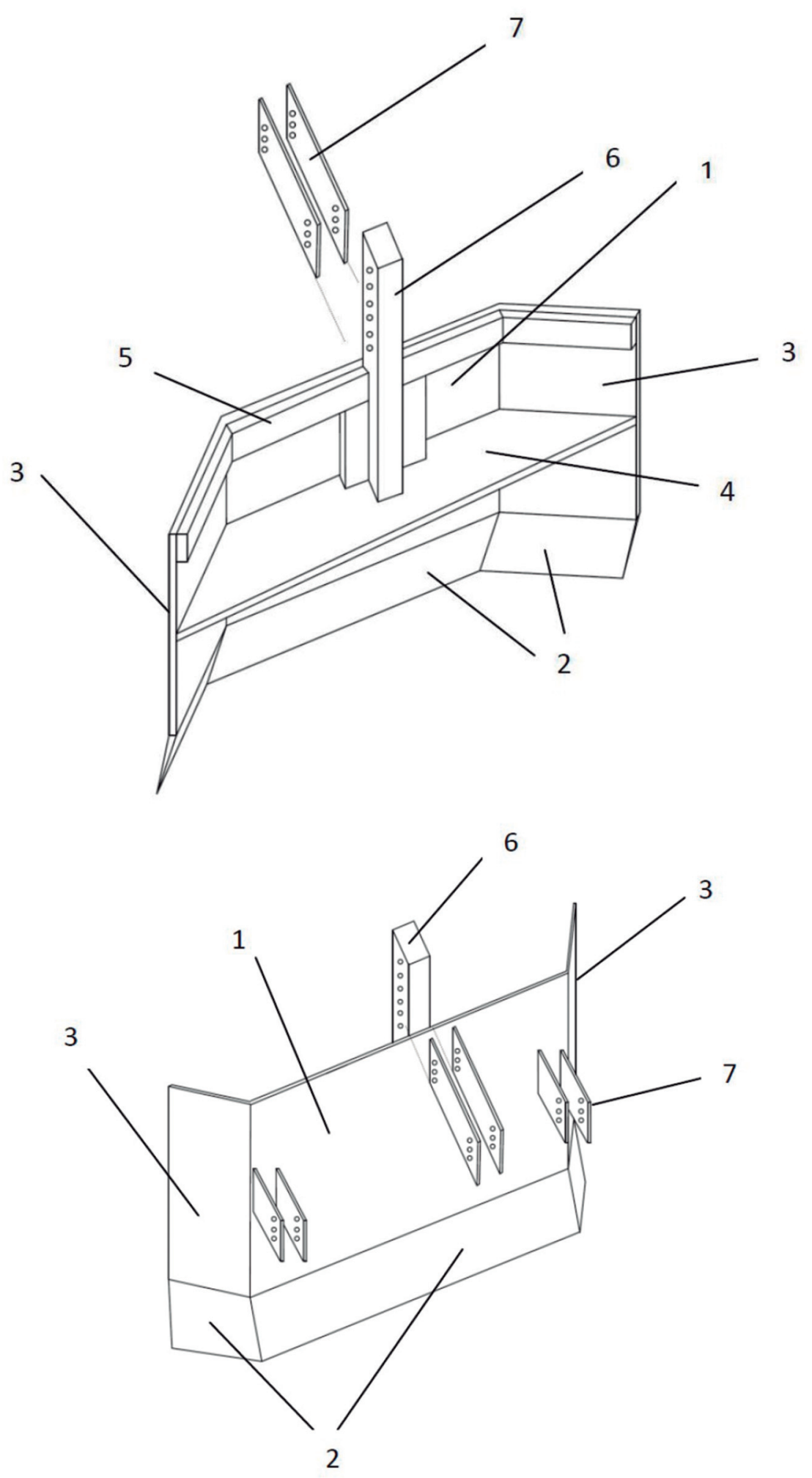

Figura 2. Vista en perspectiva posterior y anterior del apero, en la que se pueden observarse los elementos que lo componen: 1. Plancha principal. 2. Cuchillas. 3. Planchas laterales. 4. Plancha de refuerzo. 5. Chasis de barras de acero. 6. Enganche al bulldózer. 7. Enganches al tractor agrícola. 


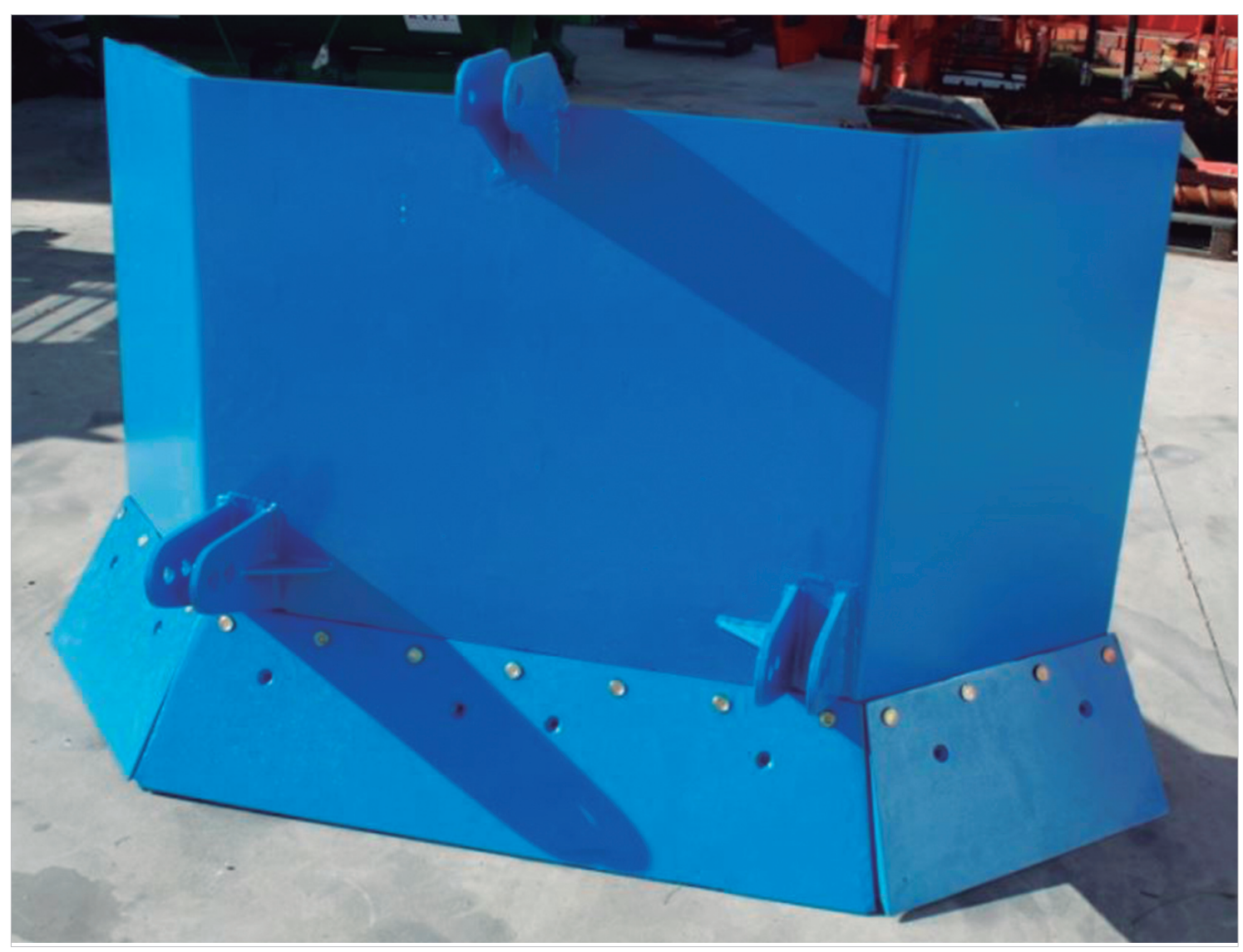

Figura 3. Vista frontal del apero

Tabla 1. Dimensiones del apero diseñado

\begin{tabular}{|c|c|c|}
\hline & MODELO a & MODELO b \\
\hline Longitud plancha principal (m) & 1.00 & 1.50 \\
\hline Altura de la plancha principal (m) & 0.80 & 0.80 \\
\hline Longitud planchas laterales (m) & 0.50 & 0.75 \\
\hline Altura de las planchas laterales $(\mathrm{m})$ & 0.80 & 0.80 \\
\hline Ángulo entre plancha principal y laterales $\left(^{\circ}\right)$ & 135 & 135 \\
\hline Altura de las cuchillas inferiores $(\mathrm{m})$ & 0.30 & 0.30 \\
\hline Ángulo de ataque de las cuchillas $\left({ }^{\circ}\right)$ & 20 & 20 \\
\hline Longitud mayor plancha de sujeción (m) & 1.71 & 2.56 \\
\hline Longitud menor plancha de sujeción (m) & 1.00 & 1.50 \\
\hline Grosor planchas de acero (mm) & 10 & 10 \\
\hline Longitud barra de enganche bulldózer (m) & 0.80 & 0.80 \\
\hline Anchura barra de enganche bulldózer (m) & 0.235 & 0.235 \\
\hline Grosor barra de enganche bulldózer (m) & 0.08 & 0.08 \\
\hline Diámetro bulón de enganche a bulldózer (mm) & 25 & 25 \\
\hline Longitud piezas de enganche tractor tercer punto $(\mathrm{m})$ & 0.377 & 0.377 \\
\hline Longitud piezas de enganche tractor brazos de tiro (m) & 0.25 & 0.25 \\
\hline Anchura barras de enganche tractor $(\mathrm{m})$ & 0.22 & 0.22 \\
\hline Grosor planchas de enganche tractor $(\mathrm{m})$ & 0.10 & 0.10 \\
\hline Peso aproximado $(\mathrm{kg})$ & 280 & 420 \\
\hline
\end{tabular}




\section{Cómo utilizar el apero}

Antes de utilizar el apero, es necesario su acoplamiento a un bulldózer o a un tractor agrícola. El tractor con el apero se sitúa en la parte superior de la ladera y va haciendo sucesivos recorridos de arriba a abajo siguiendo líneas de máxima pendiente. En el descenso del tractor, y a la distancia previamente fijada, el maquinista baja el apero clavándolo en el suelo y arrastrándolo una distancia que también ha sido fijada con anterioridad, por ejemplo $1 \mathrm{~m}$, construyendo así una microcuenca. A continuación, el maquinista levanta el apero, y sigue descendiendo por la ladera repitiendo la operación. En algunas ocasiones, con suelos compactos, puede ser necesario que el tractor dé marcha atrás y vuelva a perfilar la microcuenca. El resultado es la creación de microcuencas de forma aproximadamente semicircular en toda la superficie de la ladera, mediante la creación de unos caballones de tierra. Estos caballones sirven para detener la escorrentía superficial e infiltrarla justo aguas arriba, para conseguir así los efectos deseados de recolección de agua y oasificación. Las dimensiones de las microcuencas y la densidad de microcuencas por hectárea quedan determinadas por tres variables: separación entre líneas de máxima pendiente que recorre el tractor, separación entre las microcuencas de una misma línea y distancia de arrastre del apero.

Es necesario aclarar que el apero realiza una preparación del terreno con el fin específico de captación de escorrentía. Es decir, el apero no realiza ni surcos ni hoyos de plantación, que será preciso ejecutar posteriormente a la conformación de la microcuenca. El diseño actual del apero puede complementarse con un rejón que pueda excavar el hoyo, y así facilitar las labores de plantación.

En la construcción de microcuencas como sistemas de recolección de agua, las ventajas del apero son las siguientes:

- Es muy económico (el coste de la construcción del apero es menor de 3.000 $€$, frente a los más de $20.000 €$ que cuestan otros aperos para la conformación de microcuencas).

- El tractor trabaja, como se ha dicho, siguiendo líneas de máxima pendiente, lo que le permite trabajar con pendientes superiores al 30\% (límite de trabajo siguiendo curvas de nivel), pudiéndose alcanzar pendientes de hasta el $60 \%$.

- La ejecución de la microcuenca permite adecuar sus dimensiones y la altura del caballón al diseño fijado previamente.

- Existe la posibilidad de simular el comportamiento hidrológico de la preparación del suelo diseñada mediante el modelo hidrológico Modipé, como se ha explicado anteriormente.

- Es muy interesante en la restauración forestal de zonas áridas, semiáridas y secas subhúmedas, para combatir procesos de desertificación.

- Puede utilizarse en plantaciones de árboles con fines paisajistas y en jardines. Es especialmente interesante en parques periurbanos, donde puede ser necesario realizar una repoblación forestal en laderas de amplia superficie.

- Así mismo puede servir para el establecimiento de cultivos leñosos (olivar, viñedo, algarrobal, almendral, etc.) en las zonas climáticas indicadas donde, 
además, se sitúan numerosos países en vías de desarrollo, con necesidades de incremento en la producción agrícola en general y de alimentos en particular.

\section{Análisis hidrológico de la preparación del suelo mediante Modipé}

El modelo hidrológico Modipé permite, entre otras cosas, simular el comportamiento hidrológico de cualquier unidad sistematizada, evaluar el efecto hídrico que tienen los distintos procedimientos de preparación del suelo en repoblaciones forestales, diseñar trampas de agua con las que incrementar la infiltración y así facilitar el arraigo y primer crecimiento del repoblado en climas mediterráneos y calcular el volumen mínimo de tierras a remover en una ladera degradada para propiciar su oasificación (Martínez de Azagra, 2000; Martínez de Azagra y Mongil, 2001; Martínez de Azagra et al., 2004).

En este caso, se va a evaluar la preparación del suelo y la unidad sistematizada que crea el apero diseñado. Para ello, se han planteado cuatro casos, que se detallan en la Tab. 2 y en la Fig. 4. Estos casos son diseñados en gabinete para ser simulados mediante el modelo Modipé. No obstante, el apero puede realizar diversas sistematizaciones en función de la elección del modelo a o b del apero, la variación de la distancia de arrastre del apero, la separación de los alcorques en una línea y la separa-

Tabla 2. Casos de estudio en la evaluación de la preparación del suelo

\begin{tabular}{|c|c|c|c|c|}
\hline \multirow{2}{*}{ DATOS } & \multicolumn{4}{|c|}{ CASOS } \\
\cline { 2 - 5 } & 1 & 2 & 3 & 4 \\
\hline Modelo de apero & $\mathrm{a}$ & $\mathrm{a}$ & $\mathrm{b}$ & $\mathrm{b}$ \\
\hline $\mathrm{S}_{1}\left(\mathrm{~m}^{2}\right)$ & 1.71 & 3.42 & 2.56 & 2.56 \\
\hline $\mathrm{S}_{2}\left(\mathrm{~m}^{2}\right)$ & 1.71 & 1.71 & 2.56 & 7.68 \\
\hline $\mathrm{S}_{3}\left(\mathrm{~m}^{2}\right)$ & 2.58 & 3.87 & 0.88 & 1.32 \\
\hline $\mathrm{S}_{\mathrm{p}}\left(\mathrm{m}^{2}\right)$ & 6.00 & 9.00 & 6.00 & 11.56 \\
\hline $\mathrm{H}(\mathrm{m})$ & 0.30 & 0.30 & 0.30 & 0.30 \\
\hline $\mathrm{CAPA}(\mathrm{L})$ & 513 & 513 & 768 & 768 \\
\hline $\mathrm{N}\left(\mathrm{Pies} \mathrm{ha}{ }^{-1}\right)$ & 1667 & 1111 & 1667 & 865 \\
\hline $\mathrm{NI}, \mathrm{NR}, \mathrm{NAC}$ & 86 & 86 & 86 & 48 \\
\hline $\mathrm{P}$ & 48 & 48 & 48 & 86 \\
\hline
\end{tabular}

$\mathrm{S}_{1}=$ Área de impluvio; $\mathrm{S}_{2}=$ Área de recepción; $\mathrm{S}_{3}=$ Área de pasillos de escorrentía; $\mathrm{S}_{\mathrm{p}}=$ Área de la unidad sistematizada; $\mathrm{H}=\mathrm{Al}-$ tura del caballón de tierra; $\mathrm{CAPA}=$ Capacidad del microembalse; $\mathrm{N}=$ Densidad de plantación; $\mathrm{NI}=\mathrm{Número}$ de curva del área de impluvio; $\mathrm{NR}=$ Número de curva del área de recepción; $\mathrm{NAC}=$ Número de curva de la ladera actual; $\mathrm{P}=$ Precipitación de cálculo (aguacero de cálculo) $=48 \mathrm{~mm}$ 
ción entre líneas. Los resultados obtenidos en el análisis se muestran en la Tab. 3 y en la Fig. 5.

A partir de los resultados obtenidos, se puede decir que al tratarse en los cuatro casos de una sistematización incompleta (preparación en la que no se capta toda la escorrentía, debido a la existencia de pasillos de escorrentía), es necesario minimizar la superficie de estos pasillos para que la captación de agua sea la máxima posible. Esto

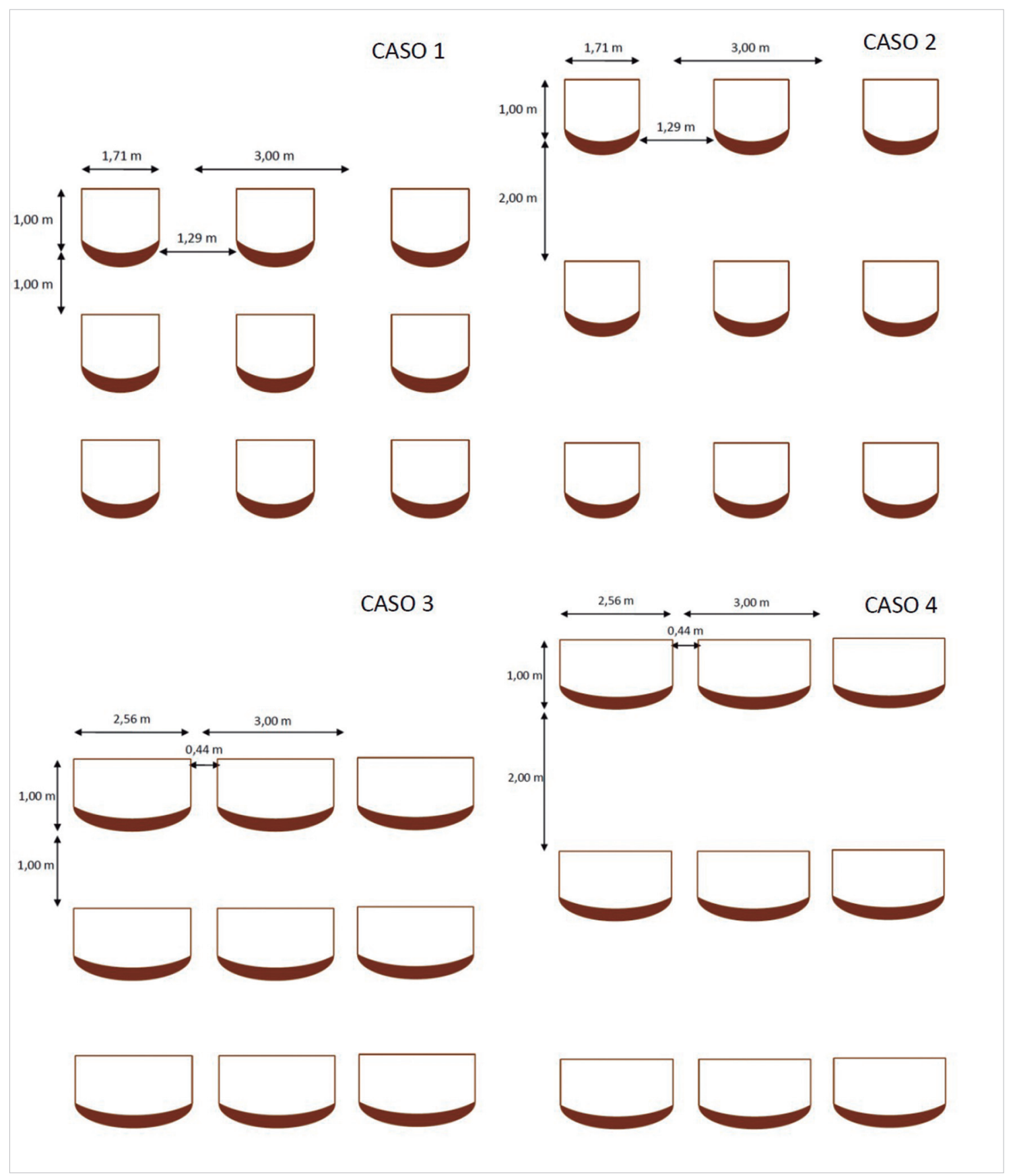

Figura 4. Diseño de las sistematizaciones en los cuatro casos analizados. 
Tabla 3. Principales resultados del análisis de la preparación del suelo con Modipé.

\begin{tabular}{|l|c|c|c|c|}
\hline \multirow{2}{*}{ VARIABLE } & \multicolumn{4}{c|}{ CASOS } \\
\cline { 2 - 5 } & 1 & 2 & 3 & 4 \\
\hline Umbral de escorrentía P0(II) ladera actual (mm) & 8.3 & 8.3 & 8.3 & 8.3 \\
\hline Umbral de escorrentía P0(II) área de impluvio (mm) & 8.3 & 8.3 & 8.3 & 8.3 \\
\hline Umbral de escorrentía P0(II) área de recepción (mm) & 8.3 & 8.3 & 8.3 & 8.3 \\
\hline Umbral de escorrentía P0(II) unidad sistematizada (mm) & 192.0 & 139.7 & 166.5 & 123.1 \\
\hline Altura mínima de las represas del alcorque (mm) & 38.9 & 58.4 & 46.5 & 26.0 \\
\hline Disponibilidad hídrica en terreno llano (precipitación) (mm) & 48.0 & 48.0 & 48.0 & 48.0 \\
\hline $\begin{array}{l}\text { Disponibilidad hídrica en la ladera actual } \\
\text { sin sistematización (mm) }\end{array}$ & 28.5 & 28.5 & 28.5 & 28.5 \\
\hline Disponibilidad hídrica en la ladera sistematizada (mm) & 48.0 & 48.0 & 48.0 & 48.0 \\
\hline Disponibilidad hídrica en el área de recepción (mm) & 67.5 & 86.9 & 75.1 & 54.5 \\
\hline $\begin{array}{l}\text { Recolección de agua en el área de recepción } \\
\text { frente a la precipitación (\%) }\end{array}$ & 70.3 & 90.5 & 78.2 & 56.8 \\
\hline $\begin{array}{l}\text { Recolección de agua en el área de recepción } \\
\text { frente a ladera actual (\%) }\end{array}$ & 118.5 & 152.5 & 131.8 & 95.6 \\
\hline $\begin{array}{l}\text { Capacidad del alcorque para retener toda la escorrentía } \\
\text { del área de impluvio (si fuese impermeable) (L) }\end{array}$ & 82.1 & 164.2 & 170.9 & 122.9 \\
\hline $\begin{array}{l}\text { Capacidad mínima del embalse para recoger } \\
\text { toda la escorrentía superficial (L) }\end{array}$ & 66.6 & 99.9 & 119.1 & 199.4 \\
\hline
\end{tabular}

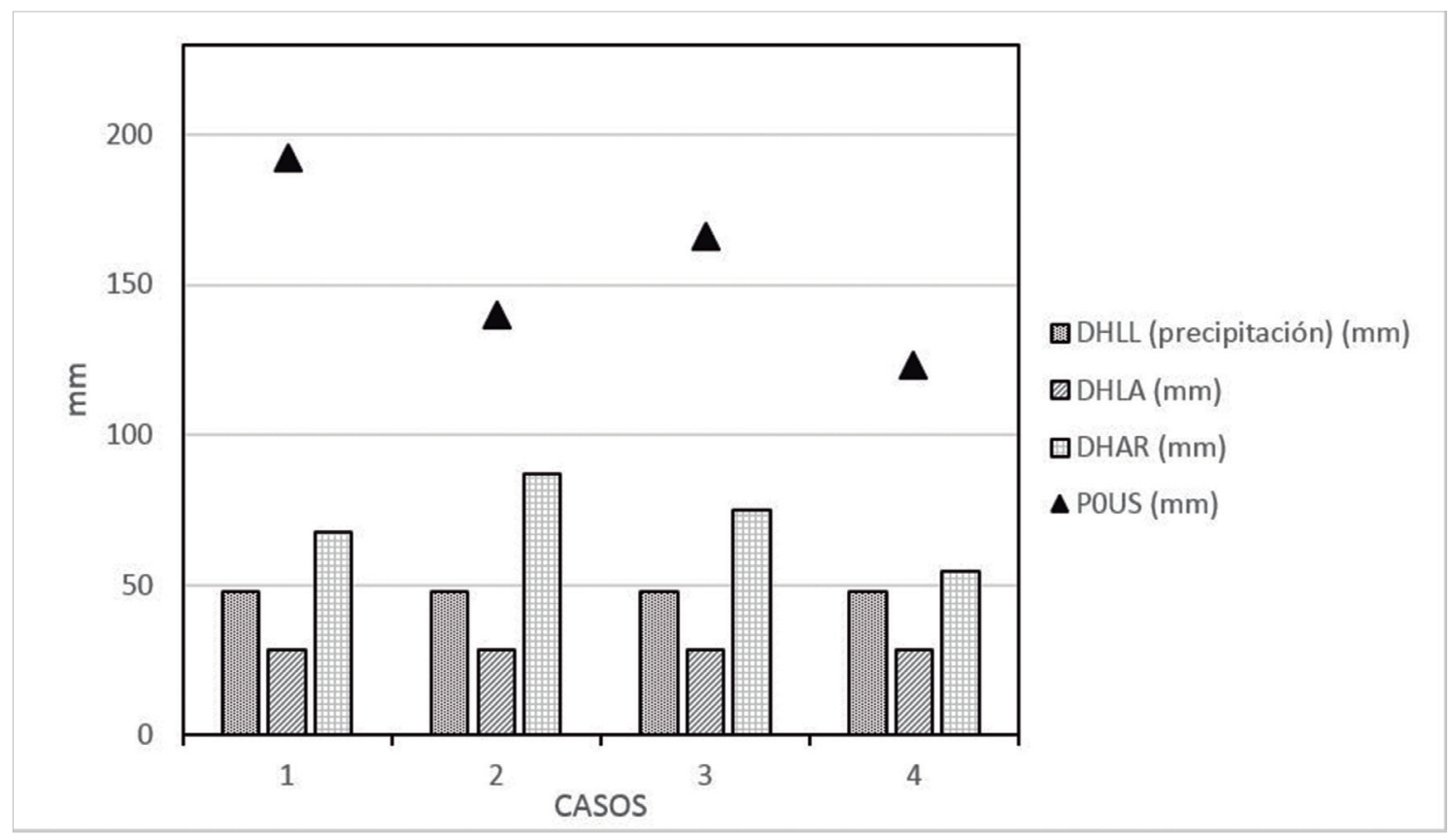

Figura 5. Disponibilidades hídricas en terreno llano (precipitación) (DHLL), en la ladera actual DHLA y en el área de recepción (DHAR) y umbrales de escorrentía en el área de recepción ( $\mathrm{P}_{0} \mathrm{US}$ ) para los cuatro casos de análisis. 
se consigue aproximando al máximo las líneas de avance del tractor, pero dejando siempre una anchura igual a la de máquina. De los casos planteados, el caso 3 es el que tiene menores pasillos de escorrentía $\left(0.88 \mathrm{~m}^{2}\right)$ frente a $\operatorname{los} 3.87 \mathrm{~m}^{2}$ del caso 2 .

Los umbrales de escorrentía de la unidad sistematizada (en condición de humedad II) son muy elevados (entre 123.1 y $192.0 \mathrm{~mm}$ ) y mucho mayores que los de la ladera actual $(8.3 \mathrm{~mm})$. Esto es de gran importancia desde el punto de vista de la economía del agua de la ladera, puesto que salvo que ocurran aguaceros de alta intensidad muy poco frecuentes, se producirá endorreísmo en la unidad sistematizada, es decir, toda el agua de lluvia y escorrentía se infiltrará dentro de ella.

Por otra parte, con la sistematización planteada se recoge una buena cantidad de agua, siempre mayor que la lluvia empleada en la simulación $(48 \mathrm{~mm})$. Si se compara la disponibilidad hídrica en la ladera actual y en el área de recepción, se puede apreciar la cuantía de la recolección de agua. En este sentido, el caso 2 es el más favorable, puesto que en el área de recepción se recogen $86.9 \mathrm{~mm}$ frente a los $28.5 \mathrm{~mm}$ de la ladera actual, es decir se obtiene una recolección de agua en el área de recepción frente a la ladera actual de un $152.5 \%$. La recolección de agua media de los cuatro casos es de $124.6 \%$. Así mismo, la recolección de agua media en el área de recepción frente a la precipitación caída es de un $74.0 \%$.

Finalmente, la capacidad mínima del alcorque para recoger toda la escorrentía superficial es, según Modipé, menor que la planteada en los cuatro casos. Es decir, para la precipitación utilizada en la simulación, el alcorque tiene el tamaño suficiente para recoger toda la lluvia y la escorrentía que se genera. Por ello, no sería necesario modificar la capacidad del alcorque dimensionada inicialmente. No obstante, la altura mínima del caballón de tierra en todos los casos es mayor que la diseñada $(30 \mathrm{~cm})$. Hay que decir en este sentido que la altura de diseño se ha fijado de forma aproximada ya que, dado que el caballón se forma con el arrastre del apero sobre el suelo, esta altura variará de unos suelos a otros. Además, aumentando la longitud de arrastre del apero se incrementará también la altura del caballón.

\section{Dónde utilizar el apero}

El apero diseñado se puede utilizar para la plantación de árboles en general, más concretamente en repoblaciones forestales; plantaciones de árboles y arbustos en otros casos, por ejemplo plantaciones ornamentales, en parques y jardines, o en restauraciones del paisaje; y en cultivos agrícolas leñosos arbóreos o arbustivos como olivares, viñedos, algarrobales, almendrales, higuerales u otros frutales. Los principales condicionantes de la utilización del apero son:

1) El clima debe ser árido, semiárido, seco subhúmedo, o sufrir una sequía estival acusada. En otros casos no sería necesaria la recolección de agua.

2) El suelo no debe ser rocoso ni excesivamente pedregoso, aunque el apero es lo suficientemente robusto como para resistir cierta pedregosidad. Los mejores suelos para el uso del apero son los sueltos, no demasiado compactados. No obstante, labores previas de remoción del suelo pueden solucionar este problema. 
3) La pendiente del terreno debe ser menor del $60 \%$, límite de trabajo de un tractor siguiendo líneas de máxima pendiente. En suelos completamente llanos no tiene sentido utilizar el apero debido a que no puede realizarse recolección de escorrentía.

4) El terreno no debe tener vegetación leñosa fuerte, salvo que se haga un desbroce previo, por ejemplo con la pala delantera de un bulldózer.

\section{Conclusiones}

El know-how aplicado al apero que se presenta en este trabajo se sintetiza en que se recoge todo el conocimiento tradicional en sistemas de recolección de agua, que crean una unidad sistematizada compuesta de un área de impluvio y un área de recepción mediante movimiento de tierras, y crean un caballón de tierra que frena, recoge e infiltra la escorrentía superficial que fluye por las laderas. De esta manera, para las precipitaciones más frecuentes de la zona, se consigue endorreísmo en la unidad sistematizada, de tal manera que toda la lluvia caída en la unidad se infiltra en ella, sin que escape en forma de escorrentía superficial. Este es uno de los principios fundamentales de la hidrología de conservación de aguas. El diseño de los enganches del apero hace posible que este se pueda acoplar tanto a un tractor agrícola como a un bulldózer. La forma de trabajo del apero, siguiendo líneas de máxima pendiente, hace que el tractor tenga mayor estabilidad y seguridad en la labor.

Lo que en los sistemas tradiciones de recolección de agua se hacía de forma manual, la excavación o movimiento de tierras, mediante el apero diseñado se realiza de manera mecanizada, lo que supone una notable mejora del rendimiento y una considerable reducción de costes. Así, gracias al diseño del apero se consigue crear una microcuenca de captación de escorrentía de forma aproximadamente semicircular, con su correspondiente caballón de tierra, donde queda retenida y se infiltra la escorrentía y la lluvia, favoreciendo la supervivencia y desarrollo de la planta que se sitúa en ella.

La preparación del suelo conseguida con el apero se simula mediante el modelo hidrológico Modipé, que permite conocer el comportamiento hidrológico del sistema de captación de escorrentía construido por el apero y mejorarlo, así como diseñar con mayor precisión la sistematización primaria de la ladera. Para los diseños de sistematización planteados, con el apero, la recolección media de agua en el área de recepción frente a la ladera actual sin restaurar es de un $125 \%$ y de un $74 \%$ frente a la precipitación caída.

\section{Bibliografía}

Martínez de Azagra, A., 2000. Principles for designing endorheic microcatchments. Third International Congress Man and Soil at the Third Millennium, Volumen I: 507-520. Martínez de Azagra, A., Mongil, J., 2001. Algunos criterios para el diseño de sistemas de 
recolección de agua en repoblaciones forestales. Actas III Congreso Forestal Español, Mesa 3: 272-277.

Martínez de Azagra, A., Mongil, J., Rojo, L., 2004. Oasification: reclamation forestry addressing desertification hazards. En: NATO-CCMS and Science Committee Workshop on Desertification in the Mediterranean Region. A security issue. Kluwer Academic Publishers.

Mongil, J., 2011. Técnicas tradicionales de recolección de agua: posibilidades de empleo en la restauración forestal. Cuadernos de la Sociedad Española de Ciencias Forestales, 32: 123-127.

Mongil, J., Martínez de Azagra, A., 2006. Diseño de repoblaciones forestales en zonas áridas: tamaño del microembalse y relación entre el área de impluvio y el área de recepción. Investigaciones Geográficas 40: 201-226.

Roose, E., 1994. Introduction à la gestion conservatoire de l'eau, de la biomasse et de la fertilité des sols (GCES). Bulletin Pédologique de la F.A.O., 70. 